\title{
Substance Abuse Treatment and Its Implications on Science and Society
}

\author{
David Christopher A* \\ Department of Behavioral Studies, USA
}

*Corresponding author: David Christopher A, Department of Behavioral Studies, California University Southern, USA, Email: christopher.david@my.calsouthern.edu

\begin{tabular}{|c|c|}
\hline ARTICLE INFO & ABSTRACT \\
\hline Received: 幽 December 30, 2019 & \multirow{7}{*}{$\begin{array}{l}\text { Substance abuse is becoming more severe as an issue despite the extensive research } \\
\text { and professional introspection of this topic. Psychology is inseparable from the under- } \\
\text { lying biological mechanisms shaped by evolution. However, many psychiatrists, thera- } \\
\text { pists, researchers, and other professional practitioners in psychology are overlooking } \\
\text { the connection the human brain has to the natural environment which it evolved along- } \\
\text { side. The loose term "drug" has created complications for using naturally occurring } \\
\text { chemicals to help restore the healthy functioning of the brain, thus the mind. Despite } \\
\text { the exorbitant resources used to develop and dispense pharmaceuticals, these chemi- } \\
\text { cal compounds lack the complete chemical structures found in nature. By studying the } \\
\text { human relationship to the natural environment in which human evolution occurred, } \\
\text { researchers can begin to understand how naturally occurring psychoactive chemical } \\
\text { compounds can help bring psychological congruence to troubled minds in the rapidly } \\
\text { developing human society observable in contemporary times. }\end{array}$} \\
\hline Published: 慧 January 10, 2020 & \\
\hline Citation: David Christopher A. Substance & \\
\hline Abuse Treatment and Its Implications on & \\
\hline Science and Society. Biomed J Sci \& Tech & \\
\hline Res 24(3)-2020. BJSTR. MS.ID.004064. & \\
\hline $\begin{array}{l}\text { Keywords: Neurological development; } \\
\text { Genetic influence; Alternative methods; } \\
\text { Drug addiction }\end{array}$ & \\
\hline
\end{tabular}

\section{Opinion}

\section{Substance Abuse Treatment and Its Implications on Science and Society}

Science, as a philosophical construct, aims to find the truth. In this sense, much of scientific endeavor remains apathetic to human existence. However, due to our self-awareness, we may be the single most significant asset the universe has to offer as a subject of study. We should treat the human brain as the focus of our pursuit. Therefore, every branch of science should involve ways to improve the world which allows us to flourish. In this paper, the author addresses substance abuse, its implications on society, and how our discoveries can help our progression of truth.

\section{Outlining Substance Abuse}

The essential features of a substance use disorder, as described by the DSM- 5 is a "cluster of cognitive, behavioral, and physiological symptoms indicating that the individual continues using the substance despite significant substance-related problems" (APA, 2013, p. 483). Also, a fundamental characteristic of this condition involves the underlying changes in neural circuitry, throughout which this paper aims to explain in more detail.

\section{The Signs and Symptoms of Substance Abuse}

All substance-using behaviors follow mostly the same pattern, excluding hallucinogens, which is relative to the argument of this paper, suggesting that some psychoactive compounds may provide treatment for substance abuse rather than be a culprit of it. Substance abuse, like most conditions in the DSM-5, lies on a spectrum from mild to severe. Its categorization includes four groups of behavioral patterns related to the use of the substance, as described below (APA, 2013). First, impaired control means an individual often takes more drugs than intended, spends excess time focusing on obtaining it, and is overall consumed by the cravings for it. Second, social impairment describes one's relationship with society, including places of work, school, or home. Such an individual will disrupt family activities or miss classes or work shifts to use drugs, causing distinct living arrangements and employment problems. Third, risky use includes using a drug despite the obvious increased risks of harm. For example, an individual with preexisting psychological problems may feel the increased urge to lash out on others or inflict self-injury. Lastly, and 
most pertinent to the neurological basis, which is the focus of this paper, is pharmacological and deals with an individual's tolerance to a substance leading to an increase in dosages to achieve the same intoxicated state, ultimately causing increased harm to other body systems, such as the liver or the central nervous system.

\section{The Development of Substance Abuse}

Despite the known adverse effects of long-term substance abuse, learned reinforcement for the drug could overpower one's cognitive control to refrain from continuing this behavior. Certainly, experimenting with a drug does not equate to an unruly death wish. Far fewer people reach an uncontrollable urge to continue this behavior than those who do; nonetheless, substance abuse causes problems across the globe Labott [1].

Reinforcement: The reinforcement mechanism responsible for destructive behaviors may seem at odds with evolutionary principles. However, one must consider an array of factors simultaneously in effect. For instance, the brain region activated by most abused drugs is the nucleus accumbens Pavuluri et al. [2]. Dopamine floods into this region resembling the natural reinforcers for the success of our species, namely, sexual contact, food satiation, and the quenching of thirst Aitken et al. [3].

Positive reinforcement: A reinforcement mechanism is most potent directly following a behavior. Therefore, if a stimulus causes a delayed response, the neurological mechanism responsible for pairing the stimulus to a response weakens accordingly. This model is consistent with studies comparing substance abuse potential and the route of administration (i.e., intravenous, smoked, oral, intranasal) Bouton [4]. Therefore, neither the damaging effects of long-term drug use nor the feeling of "coming down" has enough clout in comparison to the immediate high sought by the user.

Negative reinforcement: Just as one seeks pleasure (i.e., positive reinforcement), one seeks to eliminate displeasure (i.e., negative reinforcement). Negative reinforcement can lead to the same compulsive behaviors as positive reinforcement. For instance, those who become physically addicted to a substance will experience the symptoms of withdrawals, which is virtually the opposite of the pleasure initially sought Chen et al. [5].At this point, drug use becomes a means to counter the depressed or agitated state rather than the seeking of a pleasurable one, thus constituting negative reinforcement.

Neurological development: Drug use first strengthens the synapses in the ventral tegmental area (VTA) by producing more glutamatergic NMDA receptors, the fundamental neurological process for learning Oliva et al. [6]. Repeating substance use is initially a compulsive behavior as a result of this classical conditioning. However, after a prolonged period of use, changes in the dorsal striatum located in the basal ganglia lead to operant conditioning, which partially defines clinical substance abuse (2016).

\section{The Psychology of Substance Abuse}

Substance abuse is fundamentally a pathological pattern of behavior; psychology is the scientific study of human behavior. Therefore, understanding this framework is essential to understanding its development. As we will see, substance abuse is a learned behavior. Our behaviors interconnect with our belief systems. That is, our thoughts can reinforce our behaviors while our behaviors can reinforce our thoughts, which ultimately can bring about the addictive symptoms described in substance abuse. This section provides a brief overview of how different psychological factors play a significant role in substance abuse. Although all these factors have due implications when unraveling the complexity of substance abuse, they would require in-depth exploration of each of its own beyond the scope of this paper. Instead, the goal of this paper ultimately lies in the treatment of this psychological condition.

Genetic influences: One cannot navigate the issue at hand without acknowledging a clear role genetics plays on neural connectivity. All genetic traits have an evolutionary basis by a pure definition of what natural selection entails. Therefore, within the human population, there will always be a varying amount of any trait on a spectrum of higher to a lower prevalence. We also know that environmental factors not only play a role in an individual but on how the genes are expressed Kandel et al. [7]. Indeed, at any given moment, within a group of people, some individuals will be genetically more prone to substance abuse than others. Rather than focusing on how our evolutionary past and environmentally influenced gene expression can control our behaviors, this paper directs attention to what factors we can identify in the here-and-now.

Physiological influences: To understand the physiological urge to use drugs, one can imagine other conditions of addictive properties. For instance, the physiological mechanisms working against an individual on a diet share many of the same attributes of those battling with substance abuse Havermans [8]. Although one could abstain from substance use altogether, unlike food consumption, human and animal studies show how the underlying mechanisms can be as strong and life-threatening in either case Shulte et al. [9].

Societal influence: While the primary concern of psychological exploration involves understanding human behavior, sociology entails the understanding of the groups of people in certain regions, organizations, cultures, or families. Sociocultural factors are often challenging to undergo scientific exploration due to the complexity of human interactions and the ethical limits we have for studying human behavior. For this reason, it is not uncommon to draw theoretical understandings through animal research. One key difference between animal studies in labs, which do well to identify genetic, environmental, and neurological factors, and human studies is the role of society. For instance, although stress is widespread across the animal kingdom, social stress factors 
become acutely measurable in human studies. Factors include social anxiety, social pressure, and a strive for independence in their families or communities Sandi et al. [10].

\section{Psychotherapy and Substance-Abuse Treatment}

Preventative measures are as vital in battling substance abuse as ever, but for those who have to succumb to this condition, therapeutic interventions must address the existential present. As specialists in the field of psychological health, researchers, medical practitioners, and therapists must work together to build a framework used to treat this condition while maintaining and appreciating the differences of each unique mind. As we have only briefly explored in this paper, substance abuse stems from an array of factors varying in influence on the overall condition of everyone. Seeking a one-size-fits-all approach is ungrounded in the science we have available today. Just as there is a collection of causes leading to substance abuse, there must be a collection of methods for one's treatment. The following treatment approach acts as a guideline to tailor to an individual according to one's unique condition. Essential to all treatment plans, psychotherapy includes psychoeducation about one's condition and the uniqueness of the individual. It entails educating the client about the dynamics of any methodological intervention involved in their recovery.

Moreover, this allows family members and close friends, and even employment the information needed to support the recovery. While the nature of each plan may vary, it would be fair for one to expect a more dominant role of family therapy where strained relationships persist than one with limited close family members. Nevertheless, the inclusiveness of family is a valuable aspect of recovering Klostermann et al. [11]. Beyond education of the condition and appurtenant stigma, a treatment plan should include forms of essential healthy lifestyle practices, such as meditation, exercise, and diet David et al. [12]. Psychotherapy should follow from an integral perspective with multiple therapeutic approaches to address the various issues involved. Every component must entail methods grounded in science and introduced and applied with mindful intent. The implementation of individual treatment alongside group and family treatment yields the most success Klostermann et al. [11]. Family therapy offers support to each individual and the whole of the family. For instance, it can be an opportunity for others to receive therapeutic aid in their lives. This opportunity for family discovery aims not to find faults or to blame others. Instead, it should foster the familial bonds that underly the problems that have arisen.

\section{Psychopharmacology and Substance-Abuse Treatment}

One argument for the superiority of psychopharmacological intervention could follow the research showing evidence for the positive effects of a specific chemical compound introduced across the blood-brain barrier. On the contrary, by using chemical compounds created in labs that mimic the neurotransmitters for the neuroreceptors we intend to activate, we are ignoring the most fun- damental understanding in all of biology, namely: evolution. There is no coincidence or supernatural concept we must embrace to accept natural approaches. Instead, it is a tautological aspect of what evolution entails. For instance, the plant-based whole foods necessary for healthy human functioning are consistent with the diets of our evolutionary past David et al. [12]. Just as other lifeforms have lived in other conditions that require other diets that sustain other forms of life, humans have their unique relationship to their natural habitat. Although we indeed can and currently do manipulate our bodies and the environment in ways consistent with human functioning, it is a fallacy to regard our human connection to nature as unscientific to explore and apply to healthy human functioning.

The current advancement in neuroscience overwhelms researchers with new perspectives and unexplored pathways Marazziti et al. [13]. However, with our broadening view comes many unanswered questions. No matter how much the brain resembles a hardwired program, we must not fall subject to treating it as merely that. Though overshadowing the recent past, our understanding of neural connectivity is insufficient to make sweeping claims about the long-term effects of altering these mechanisms, no matter how mechanical we perceive them to be.

\section{Alternative Methods of Substance-Abuse Treatment}

Scientific research has proved beneficial across medicine, physical health, and mental well-being. However, the reductionist nature of scientific discovery creates blind spots in our wholeworld view. For example, efficacious science requires us to eliminate confounding factors that could be responsible or otherwise relevant to the intricacies we study. Unfortunately, this approach has fragmented our understanding into separate blocks of knowledge and even entirely different disciplines, which often do not overlap and cause conflicts when they do. Considering the complexity of the brain and the neural connectivity, which can change and reorder itself, we must reevaluate some of our methods Sala Llonch et al. [14]. For instance, is it scientifically rational to administer pharmaceutical interventions to treat the brain based on the success of a single biomarker? of course, when a single biomarker makes the difference in preventing a potentially fatal event, it does, but this most often is not the case. As we know, long-term drug use can cause changes to the brain on a synaptic level Wetherill et al. [15]. However, neural circuiting is much more complex than the sum of all synaptic potentials. In effect, we now know neural circuiting can be rerouted and strengthened along with new circuits (2013).

This brain plasticity, once discountenanced in science, implicates more than just changing our views about this area. We must rethink the scientific studies that used this premise to draw their conclusions. Psychopharmacological intervention might be the best way to produce and deliver a surplus of neurotransmitters for some regions of the brain, but it concurrently activates a plethora of other body systems with receptors for those neurotransmitters 
with varying effects we have not fully identified David et al. [16]. We could make the logical and scientifically grounded assumption that whatever we are looking for has evolved alongside us, just like the air, food, and water we need to survive. Furthermore, it is reasonable to suggest that these chemical compounds are already known to humankind. After all, pharmaceutical companies create compounds to mimic those existing in nature. It is true that part of scientific discovery is identifying the mechanisms behind it, but we are underway this ambitious endeavor. We have yet to unlock these current mysteries; thus, we must not make sweeping claims about the entire systems based on reductionist views. After all, a lack of understanding of the mechanisms does not warrant abandoning the further investigation. Instead, this should be the catalyst to advance its research.

\section{A Promising Prospect of Future Discoveries}

At present, one of the most studied and formidable prospects of using naturally occurring chemical compounds is the ayahuasca drink originating from South American shamanism. This psychoactive compound is only one example of thousands of naturally occurring chemical compounds that should employ further investigation of using ancient methods for restoring human health. Systematic literature reviews have revealed behavioral parameters and biochemical improvements in all of five animal studies and all but one of five human studies Nunes et al. [17]. Even when researchers set out to implicate the adverse effects of ayahuasca in therapeutic use, the side effects are minor in comparison to standard pharmaceutical intervention methods Hamil et al. [18]. It is false to claim that our natural environment possesses miraculous cures to all the world's problems. Moreover, it has always been a symbiotic relationship. Our bodies have evolved to interact with the elements around us, just as the elements around us have evolved to interact with us. This concept goes back to the beginning of life. It is scientifically absurd to overlook this fact and it undermines evolution, the most encompassing theoretical framework ever discovered in biology and perhaps all of science.

\section{Notable Considerations}

Despite these promising results on a condition with limited success in treatment methods, there are still serious cautions to consider. For instance, due to the powerful effect of reorganizing neural circuits, this method could be harmful to younger individuals whose brains are still developing or the fetus a pregnant woman's womb Labate [19]. Furthermore, this chemical compound and many others will impair driving or operating machinery, implying the need for a controlled environment for its therapeutic use Pic Taylor [20]. In addition to its positive publicity of curing the addictive symptoms of substance abuse, research might send the message to the public that using these substances are safe for all users Cruz et al. [21]. Indeed, the proper education of the public is necessary for our advancement of researching these potentially valuable but still powerful substances. We need to carry out more studies, which causes some difficulty in how to progress. Researchers must have adequate access to administer clinical trials without the fear of facing legal penalties, while it needs to be inaccessible to the general public where it could get in the hands of children or those with preexisting mental disorders. For example, most ayahuasca studies have focused on drug addiction and depression, and assuming these positive results will carry over to other conditions is understudied. Furthermore, there is a reason to believe that those with psychotic symptoms, schizophrenia, or certain behavior disorders may have adverse reactions to these compounds worsening their conditions dos Santos [22]. These are the questions needed to further our exploration and discovery of the implications of what alternative therapeutic interventions have on mental health.

\section{Conclusion}

The current advancement in neuroscience overwhelms researchers with new perspectives and unexplored pathways. Ultimately, substance abuse is only one psychological condition addressed in this paper. Nevertheless, it is an excellent example of the current problems our world faces. At this time, and progressively for the last century, this problem has worsened. We must open our minds to the innovative research with which advances in neuroscientific technology has provided us, even if it takes us back to studying ancient human wisdom. Time, effort, and resources are needed to take on this endeavor. However, the potential benefits to society are immeasurable. By establishing a neurological health reform that uses the natural plants which evolved alongside us, we could eliminate the stronghold of pharmaceutical companies on the health of a nation. Furthermore, the cultivation and preparation of these plants would provide farmers and agriculturists around the world new prospective visions. Most of all, a struggle for adequate health care could be replaced with affordable and accessible treatment plans that have been tried and tested with the same scientific inquiry we see in pharmaceutical research.

\section{References}

1. Labott SM (2019) Substance use and abuse. In Health psychology consultation in the inpatient medical setting. Washington, DC: American Psychological Association pp. 183-197.

2. Pavuluri M, Volpe K, Yuen A (2017) Nucleus accumbens and its role in reward and emotional circuitry: A potential hot mess in substance use and emotional disorders. AIMS Neuroscience 4(1): 52-70.

3. Aitken TJ, Greenfield VY, Wassum KM (2015) Nucleus accumbens core dopamine signaling tracks the need-based motivational value of foodpaired cues. Journal of Neurochemistry 136(5): 1026-1036.

4. Bouton M (2014) Why behavior change is difficult to sustain. Preventive Medicine 68(1): 29-36.

5. Chen P, Huang M, Lai Y, Chen P, Liu H (2014) Serum brain-derived neurotrophic factor levels were reduced during methamphetamine early withdrawal. Addiction Biology 19(3): 482-485.

6. Oliva I, Wanat MJ (2016) Ventral tegmental area afferents and drugdependent behaviors. Frontiers in Psychiatry 7(30). 
7. KandelD, Kandel E (2014) The gateway of substance abuse: Development biological and societal perspectives. ACTA Paediatrica 104(2): 130-137.

8. Havermans RC (2013) Pavlovian craving and overeating: A conditioned incentive model. Current Obesity Reports 2(2): 165-170.

9. Shulte EM, Yokum S, Potenza MN, Gearhardt AN (2016) Neural systems implicated in obesity as an addictive disorder: from biological to behavioral mechanisms. Progress in Brain Research 223(1): 329-346.

10. Sandi C, Haller J (2015) Stress and the social brain: Behavioural effects and neurobiological mechanisms. Nature Reviews Neuroscience 16(1): 290-304.

11. Klostermann K, O’Farrell TJ (2013) Treating substance abuse: Partner and family approaches. Social Work in Public Health 28(3-4): 234-247.

12. David CA, Coan PG (2018) Depression and the ending of depression: Tradition, science and subjectivities. Life Research 1(2): 68-72.

13. Marazziti D, Presta S, Baroni S, Silvestri S, Dell'Osso L (2014) Behavioral addictions: A novel challenge for psychopharmacology. CNS Spectrums 19(6): 486-495.

14. Sala Llonch R, Bartrés Faz D, Junqué C (2015) Reorganization of brain networks in aging: a review of functional connectivity studies. Frontiers in Psychology 6(663).

15. Wetherill R, Tapert SF (2013) Adolescent brain development, substance use, and psychotherapeutic change. Psychology of Addictive Behaviors 27(2): 393-402.

ISSN: 2574-1241

DOI: 10.26717/BJSTR.2020.24.004064

David Christopher A. Biomed J Sci \& Tech Res

(C) This work is licensed under Creative

Submission Link: https://biomedres.us/submit-manuscript.php
16. David CA, Pardo N (2018) Reconsidering Psychopharmacology: The Logical Approach. Journal of Traditional Medicine \& Clinical Naturopathy 7(1): 1-4.

17. Nunes AA, dos Santos RG, Osório FL, Sanches RF, Crippa JAS, et al. (2016) Effects of ayahuasca and its alkaloids on drug dependence: A systematic literature review of quantitative studies in animals and humans. Journal of Psychoactive Drugs 48(3): 195-205.

18. Hamill J, Hallak SM, Dursun GB (2019) Ayahuasca: Psychological and physiologic effects, pharmacology and potential uses in addiction and mental illness. Current Neuropharmacology 17(2): 108-128.

19. Labate BC (2011) Consumption of ayahuasca by children and pregnant women: Medical controversies and religious perspectives. Journal of Psychoactive Drugs 43(1): 27-35.

20. Pic Taylor A, da Motta LG, de Morais JA, Junior WM, Santos AF, et al. (2015) Behavioural and neurotoxic effects of ayahuasca infusion (Banisteriopsis caapi and Psychotria viridis) in female wistar rat. Journal of Behavior Processes 118(1): 102-110.

21. Cruz JI, Nappo SA (2018) Is ayahuasca an option for the treatment of crack cocaine dependence? Journal of Psychoactive Drugs 50(3): $247-$ 255.

22. dos Santos RG, Bouso CB, Hallak JEC (2017) Ayahuasca, dimethyltryptamine, and psychosis: a systematic review of human studies. Therapeutic Advances in Psychopharmacology 7(1): 141-157.

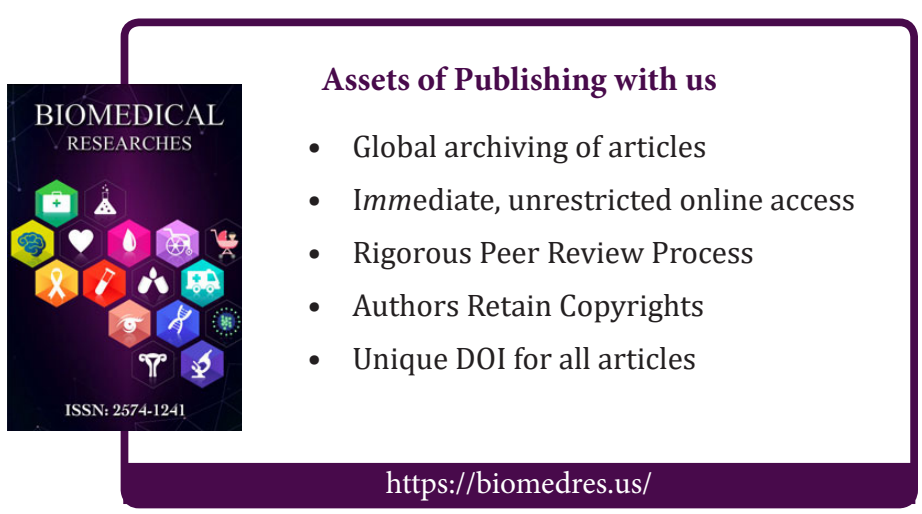

